\title{
MAKING CURVED INTERFACES STRAIGHT IN PHASE-CHANGE PROBLEMS
}

\author{
MARIO STORTI*, LUIS A. CRIVELLI* AND SERGIO R. IDELSOHN ${ }^{\dagger}$ \\ Mechanical Laboratory of INTEC, (Instituto de Desarrollo Tecnologico para Ia Industria Quimica), Universidad Nacional \\ del Litoral and CONICET, P.O. Box 91,3000 Santa Fe, Argentina
}

\begin{abstract}
SUMMARY
This paper presents a method for straightening curved interfaces arising in phase-change problems. The method works on isoparametric finite elements, performing a second transformation which maps the master element onto a new one in which the interface looks like a straight line. This allows using the current Gaussian integration technique for squares to evaluate the integrals over each phase. The method provides a better estimation of the contribution of latent heat effects to the residual vector, compared to those obtained by using the assumption that the interface is straight. Appropriate guidelines for solving the non-linear system of equations arising in this kind of problem are also given. Several numerical examples are presented to show the performance of the method.
\end{abstract}

\section{INTRODUCTION}

During recent years very much interest has been devoted to the numerical solution of phase-change problems. This phenomenon takes place in many processes of technological interest, such as soil freezing, the steel and glass industries, thermal protection of heated devices, nuclear safety analysis and many others. ${ }^{1,2}$ This problem is characterized by a geometric-type non-linearity due to the moving boundaries of the regions where the heat transfer equation must be solved. ${ }^{3}$ Regarding this fact, many authors have developed algorithms which track the position of the moving front. There are a number of works which use a deforming grid formulation. ${ }^{4-6}$ In these methods the interface always lies on one side of some determined elements. The grid moves to adapt to the displacements of the interface, remaining the same elements in the solid and liquid phases. As an alternative to reduce the number of degrees of freedom, $\mathrm{O}^{\prime} \mathrm{Neill}^{7}$ discretizes $^{\circ}$ only the boundary of each phase, using Green functions to eliminate the volume integrals. This boundary element method is applied to the quasisteady form of the heat transfer equation (low Stefan number) in materials where the thermal coefficients are not temperature-dependent. Although tracking methods are a 'natural' approximation to this problem, there are a number of drawbacks that induce using fixed domain methods. Among them one can mention that they need starting solutions and that they cannot cope with appearing/disappearing phases and multiple or no-smooth interfaces.

Fixed domain methods use weak formulations ${ }^{8,9}$ and implicitly contain the interface moving condition in the integrated form of the heat transfer equation. Atthey ${ }^{10}$ uses the enthalpy as the

\footnotetext{
* Research Fellow from CONICET.

† Scientific and Technological Research Staff Member of CONICET.
}

0029-5981/87/020375-18\$09.00

(C) 1987 by John Wiley \& Sons, Ltd.

Received 12 June 1985

Revised 6 June 1986 
nodal unknown and an explicit scheme for the time integration. Once the enthalpy is determined for a given time step, he obtains the temperature applying the inverse enthalpy-temperature relation. Comini et al. ${ }^{11}$ replace the effect of the latent heat by an equivalent hoat capacity valid in a narrow range of temperatures around the melting temperature. In this way, this equivalent heat capacity method removes the discontinuity at the phase-change front, allowing the use of the temperature as the main unknown. However this class of method has the drawback that the time step used must be small enough to prevent the interface from advancing more than one element per time step, otherwise the algorithm loses part of the latent heat involved. Meyer ${ }^{2}$ instead, splits the interface into a phase-change region within which the enthalpy varies linearly with the temperature. But when this mushy region moves between two nodes, the algorithm does not realize its motion. A way to circumvent this objection is to use a large regularization parameter so that the phase-change region contains several elements. Hence the solution is strongly dependent on the size of the regularization parameter whose proper magnitude is unknown a priori. Rolph and Bathe ${ }^{13}$ and Roose and Storrer ${ }^{14}$ use a fictitious heat source applied to those elements which are changing phase, until such elements are completely melted. The features of these algorithms resemble those of the enthalpy-based methods. Blanchard and Fremond ${ }^{15}$ integrate the heat transfer equation with respect to time, defining a freezing index as the main unknown. Furthermore they regularize the enthalpy to treat mushy regions.

To avoid the necessity of an explicit smoothing in the temperature formulation, the use of discontinuous finite elements ${ }^{16}$ has been proposed. In this way, it is possible to accurately integrate the latent heat contribution to the equilibrium equation. When an interface traverses

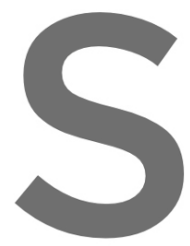
an element, it is split each part. The discontin Poisson equation in specified internal bou References 16 and $1^{\prime}$ practical cases, as when using tihear quadrilateral finite elements,
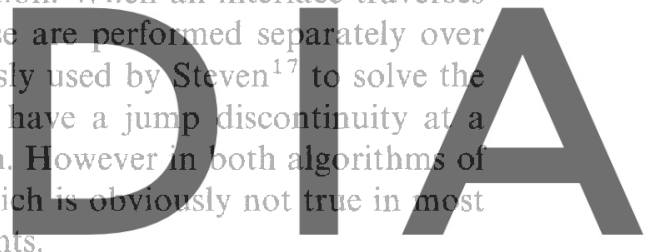

In this paper, the two-dimensional phase-change problem is analysed. The discretization

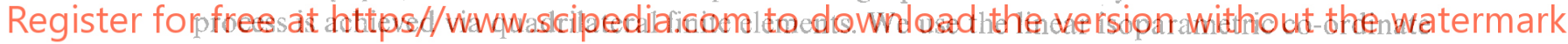
transformation to evaluate the element matrices and a second mapping in those elements traversed by an interface to obtain a new representation of the elements in which the interface looks like a straight line. Then the integrals can be accurately calculated using numerical integration for triangles or quadrilaterals. Finally, the solution of the non-linear system of equations is briefly studied. We use a modified Newton scheme to improve convergence and avoid numerical instability of the iterative scheme. Several numerical examples are presented to show the behaviour of the method. It is worth mentioning that this procedure may be straightforwardly applied to other physical problems where there can be elements with an abrupt internal interface.

\section{GOVERNING EQUATIONS AND FINITE ELEMENT FORMULATION}

The heat conduction process is governed by the partial differential equation

$$
\nabla^{\mathrm{T}} k \nabla T+Q=\rho \frac{\partial H}{\partial t}
$$

in a domain $\Omega$, with appropriate boundary and initial conditions. In equation (1) $k$ is the thermal conductivity, $T$ the temperature, $Q$ the internal heat source, $\rho$ the density, $H$ the specific enthalpy and $t$ the time.

For phase-change problems the enthalpy has a jump discontinuity at the melting temperature 
$T_{\mathrm{m}}$, equal to the latent heat content per unit volume $L$. Therefore $H$ attains a discontinuity at the free boundary. The integral weak form ${ }^{8,16}$ of equation (1) takes into account this discontinuity and is the basis for a finite element analysis. ${ }^{18}$ Integrating equation (1) weighted by some functions $N_{i}(\mathbf{x})$ and applying an $\alpha$-method ${ }^{19}$ for the time derivative of the enthalpy, we have

$$
\begin{aligned}
& -\int_{\Omega} \nabla N_{i}(\mathbf{x}) k\left(T_{n+\alpha-1}\right) \nabla T_{n+\alpha-1} \mathrm{~d} V-\int_{\Omega} N_{i}(\mathbf{x}) \frac{H_{n+\alpha-1}-H_{n-1}}{\alpha \Delta t} \mathrm{~d} V \\
& \quad+\int_{\Omega} N_{i}(\mathbf{x}) Q_{n+\alpha-1} \mathrm{~d} V+\int_{\partial \Omega_{q}} N_{i}(\mathbf{x}) q_{n+\alpha-1} \mathrm{~d} S=0
\end{aligned}
$$

with $0 \leqslant \alpha \leqslant 1$. In equation (2), $q$ is the prescribed heat flux on the portion $\partial \Omega_{q}$ of the boundary and the subscript $n$ denotes values evaluated at specific times of the time interval. We shall concentrate here on implicit methods, more precisely when $\alpha=1$. Equation (2) may be rewritten in a compact form as

$$
\frac{1}{\Delta t} i_{j}+f_{j}-y_{j}=0
$$

where $f_{j}$ is the heat conduction contribution to node $j, g_{j}$ is the external flux and $i_{j}$ is the nodal enthalpy. Applying Galerkin's method, that is using the same weighting functions as shape functions for the temperature discretization, i.e.
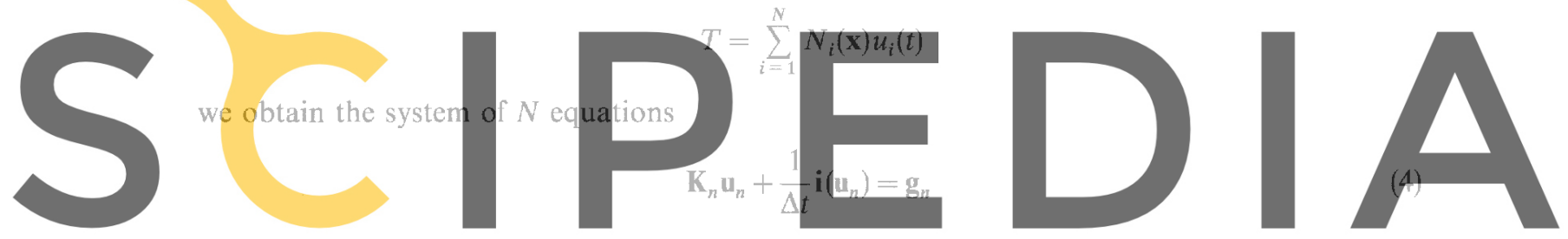

where $\mathbf{K}$ is the stiffness matrix, i is the enthalpy vector, $\mathbf{g}$ is the force vector and $\mathbf{u}$ is the vector

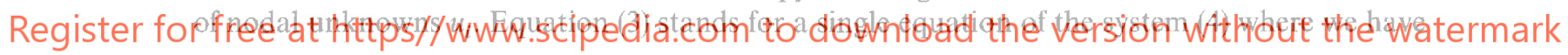

$$
\begin{aligned}
{[\mathrm{K}]_{i j} } & =\int_{\Omega} \nabla N_{i} k \nabla N_{j} \mathrm{~d} V \\
{[\mathbf{i}]_{i} } & =\int_{\Omega} N_{i} H_{n} \mathrm{~d} V \\
{[\mathbf{g}]_{i} } & =\int_{\Omega} N_{i} Q_{n} \mathrm{~d} V+\int_{\Omega} \frac{1}{\Delta t} N_{i} H_{n-1} \mathrm{~d} V+\int_{2 \Omega_{q}} N_{i} q_{n} \mathrm{~d} s
\end{aligned}
$$

When the interface traverses an element, the enthalpy becomes discontinuous inside it and equation (5b) cannot be adequately integrated using a numerical technique such as the Gaussian rule. ${ }^{16}$

Applying the linear isoparametric transformation ${ }^{18}$ to the two-phase element of Figure 1(a), i.e.

$$
\mathbf{x}=\sum_{i=1}^{N} N_{i}(\xi, \eta) x_{i}
$$

where $N_{i}, i=1, \ldots, N$ are the same linear shape functions uscd for the discretization of the temperature and $x_{i}$ are nodal co-ordinates, this element is mapped onto a master which in the $(\xi, \eta)$ plane looks as in Figure 1(b). Then the integrals (5) can be performed in the master. The nodal enthalpy contribution from equation $(5$ b) reads as 


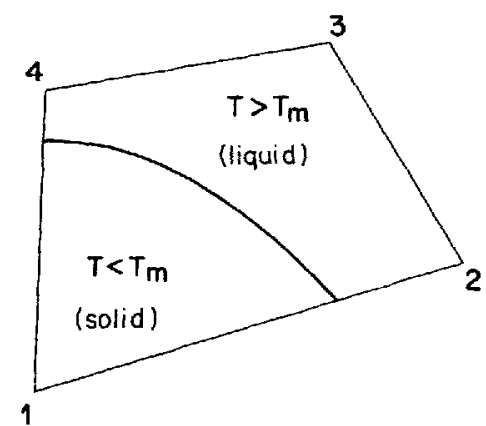

(a)

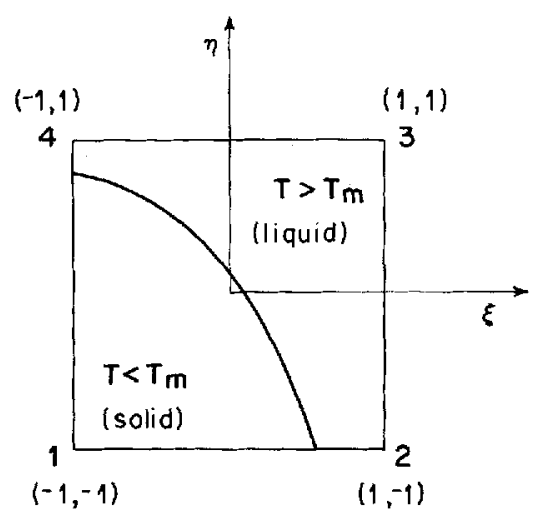

(b)

Figure 1. A typical two-phase element (a) and its isoparametric representation (b)

$$
[\mathrm{i}]_{j}=\int_{-1}^{1} \int_{-1}^{1} N_{j}(\xi, \eta) H_{\left(T_{(x)}\right)} \operatorname{det} \mathrm{J} \mathrm{d} \xi \mathrm{d} \eta
$$

where $\mathbf{J}$ is the Jacobian of the isoparametric transformation.

In order to integrate the discontinuity accurately, equation (6) may be evaluated separately in both regions, frozt

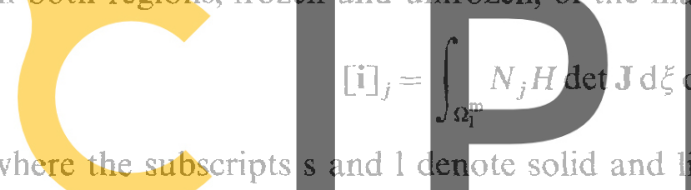

master element. Considering
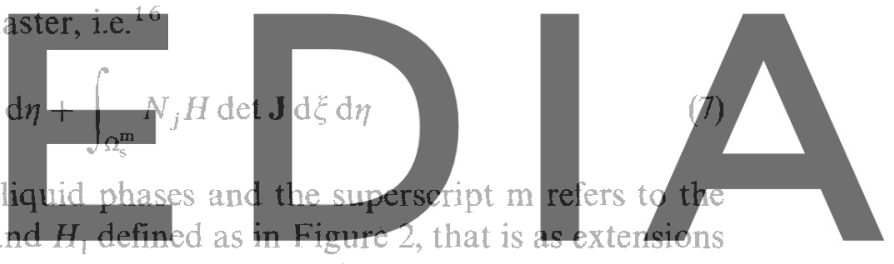

of the smooth portions of $H$, equation (7) may be written in the two following alternative ways

Register for free at https//www.scipedia.com to download the version without the watermark

$$
\begin{aligned}
& {[i]_{j}=\int_{-1} N_{j} H_{1} \operatorname{det} J \mathrm{~d} \xi \mathrm{d} \eta+\int_{\Omega_{\mathrm{s}}^{\mathrm{m}}} N_{j}\left(H_{\mathrm{s}}-H_{1}\right) \operatorname{det} \mathbb{J} \mathrm{d} \xi \mathrm{d} \eta} \\
& {[\mathbf{i}]_{j}=\int_{-\mathbf{1}}^{1} \int_{-1}^{1} N_{j} H_{\mathrm{s}} \operatorname{det} \mathbf{J} \mathrm{d} \xi \mathrm{d} \eta+\int_{\Omega_{1}^{\mathrm{m}}} N_{j}\left(H_{1}-H_{\mathrm{s}}\right) \operatorname{det} \mathbf{J} \mathrm{d} \xi \mathrm{d} \eta}
\end{aligned}
$$

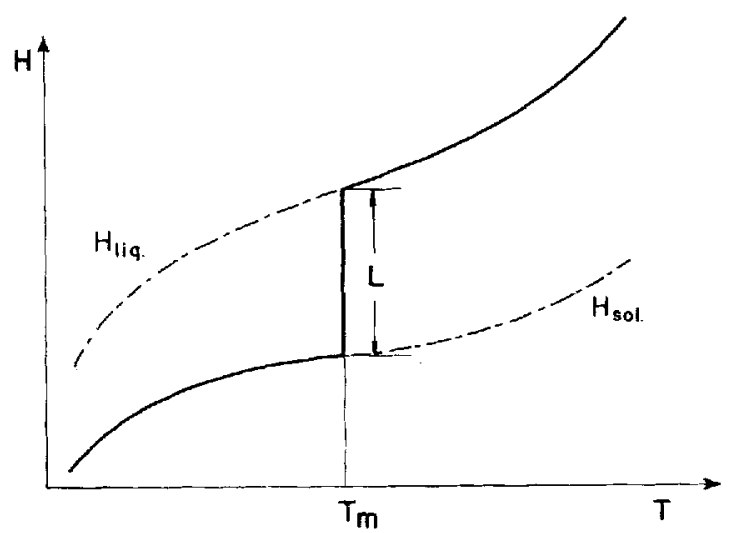

Figure 2. Extended enthalpy functions for the solid and the liquid 
As the integrands in each of the first right-hand side terms in equations (8) are smooth and the integration domain is a square, the problem is reduced to evaluating the integrals of the difference between $H_{\mathrm{s}}$ and $H_{1}$ in any of the regions into which the master element is divided.

\section{THE SPECIAL ELEMENT}

Since the solid and liquid regions have arbitrary curved boundaries, there are no simple numerical integration schemes to evaluate the second integrals in equation (8). The solution proposed in this paper is to find a mapping of the $(\xi, \eta)$ plane onto another $\left(\xi^{\prime}, \eta^{\prime}\right)$, in which these regions are transformed into a triangle or a quadrilateral, according to whether the interface cuts two adjacent or two opposite sides, as shown in Figure 3. Once this transformation is obtained (and may be easily inverted), the problem is solved by integrating in the hatched area of the elements of Figure 3. A first trial may be the mapping $(\xi, \eta) \rightarrow(\xi, T)$ where in each element $T$ is given by

$$
T=N_{1} u_{1}+N_{2} u_{2}+N_{3} u_{3}+N_{4} u_{4}
$$

Figure 4 shows the result of this transformation. It is trivial that under this mapping, the interface is a straight line $T=$ constant, parallel to the $\xi$ axis. Furthermore all the sides of the quadrilateral remain straight because at $\xi$ or $\eta$ constant, $T$ is a linear function of the remaining variable. However, a problem arises when $T_{1}=T_{4}$ or/and $T_{2}=T_{3}$ because this transformation is not one to one, but the element is mapped onto a triangle or a line in the $(\xi, T)$ plane. This mapping may be modified to override this drawback. Let us note that the temperature may be
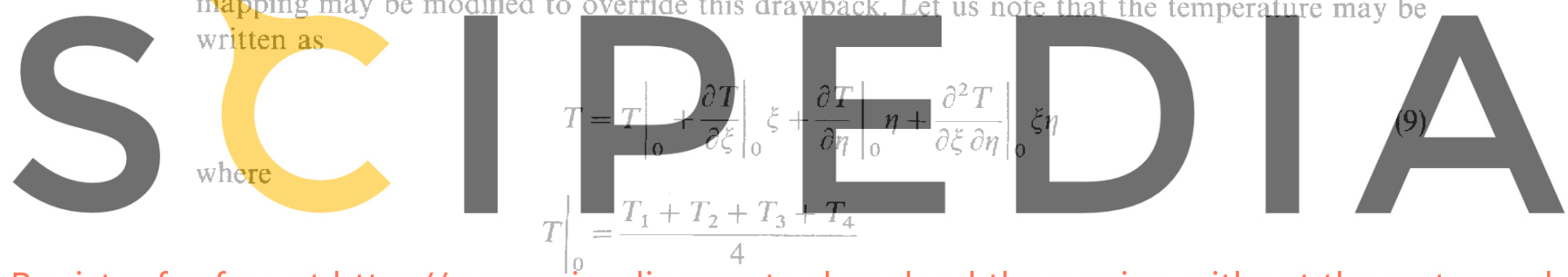

Register for free at https//www.scipedia.com to download the version without the watermark
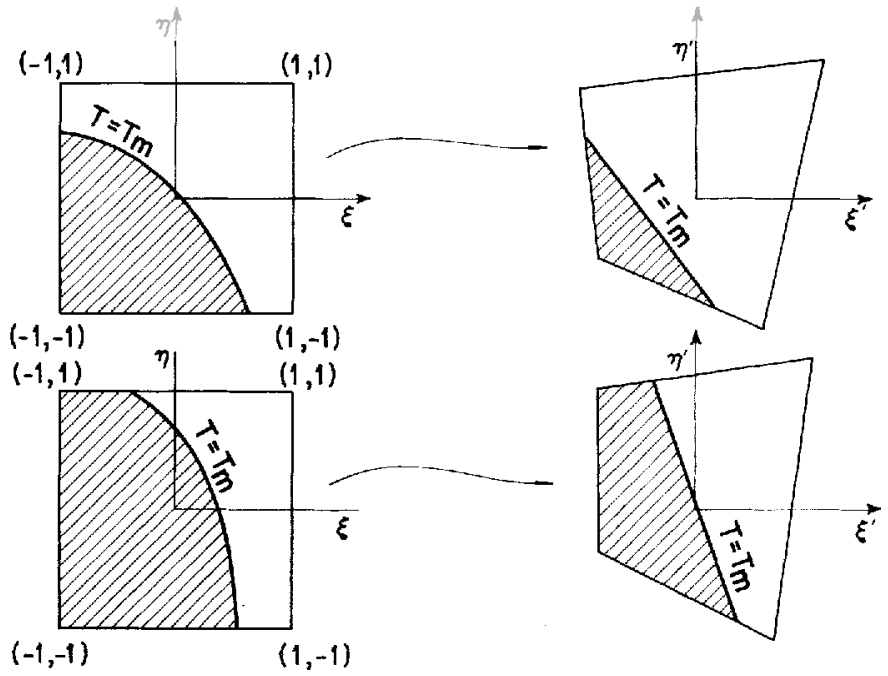

Figure 3. Transformation of the two-phase master element to a representation where the interface looks like a straight line 

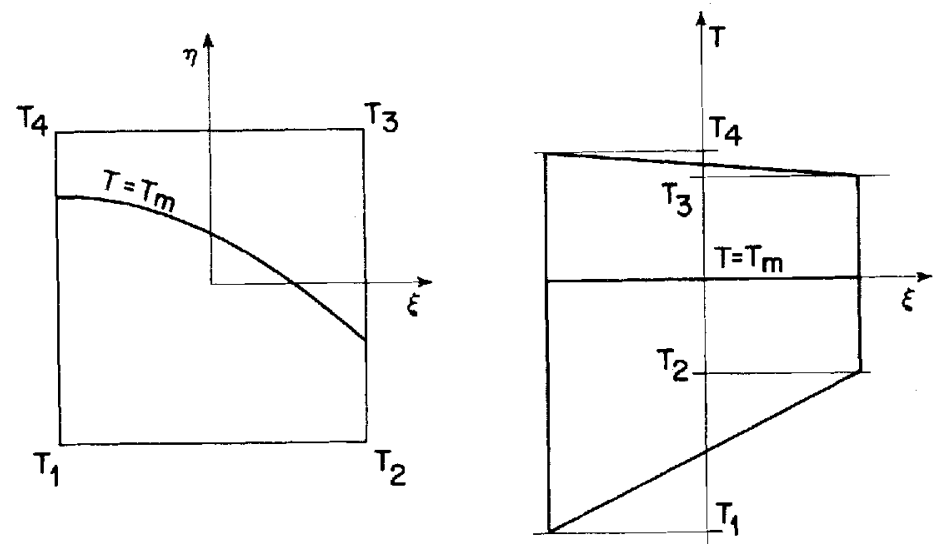

Figure 4. Transformation of the two-phase master element under the mapping $(\xi, \eta) \rightarrow(\xi, T)$

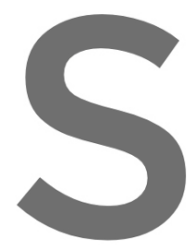

$\left.\frac{\partial T}{\partial \xi}\right|_{0}=\frac{T_{2}+T_{3}-T_{1}-T_{4}}{4}$
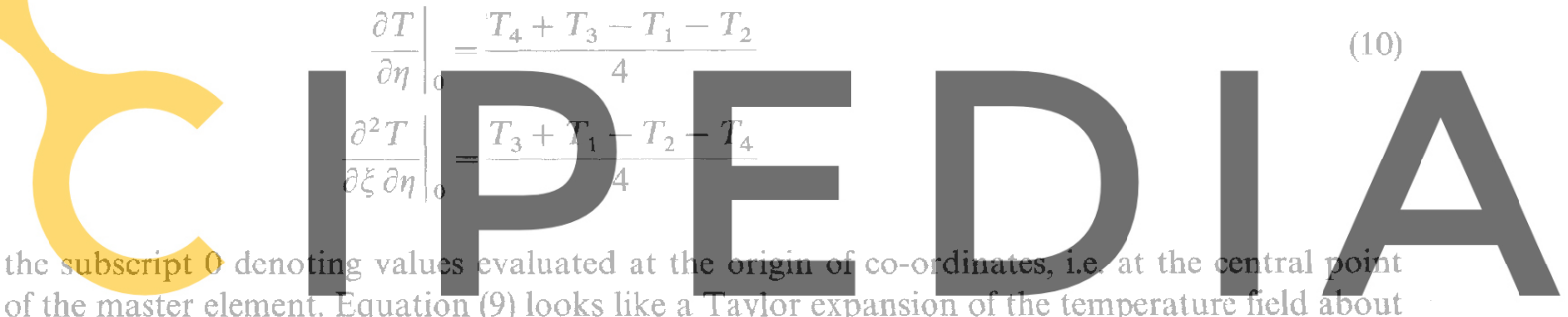

the origin of the master's co-ordinate system. This expansion has only four non-zero terms since

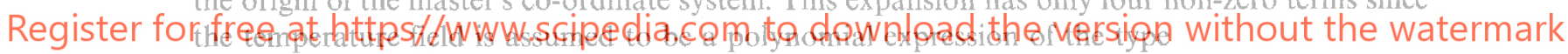

$$
T=a_{0}+b \xi+c \eta+d \xi \eta
$$

inside each element. From equation (10) we can see that the quantities evaluated at the middle point of the master element can be viewed as average quantities over the master element, i.e.

$$
\begin{gathered}
\left.T\right|_{0}=\frac{1}{4} \int_{-1}^{1} \int_{-1}^{1} T \mathrm{~d} \xi \mathrm{d} \eta=\bar{T} \\
\left.\frac{\partial T}{\partial \xi}\right|_{0}=\frac{1}{4} \int_{-1}^{1} \int_{-1}^{1} \frac{\partial T}{\partial \xi} \mathrm{d} \xi \mathrm{d} \eta=\frac{\partial T}{\partial \xi}
\end{gathered}
$$

and so on. Hereafter we shall use a barred symbol to denote such values. Therefore a second trial reads

$$
\left[\begin{array}{l}
\xi \\
\eta
\end{array}\right] \rightarrow\left[\begin{array}{l}
T \\
V
\end{array}\right]=\left[\begin{array}{c}
\bar{T}+\frac{\overline{\partial T}}{\partial \xi} \xi+\frac{\overline{\partial T}}{\partial \eta} \eta \frac{\overline{\partial^{2} T}}{\partial \xi \partial \eta} \xi \eta \\
-\frac{\partial T}{\partial \eta} \xi+\frac{\partial T}{\partial \xi} \eta
\end{array}\right]
$$


The new coordinate system may be written as

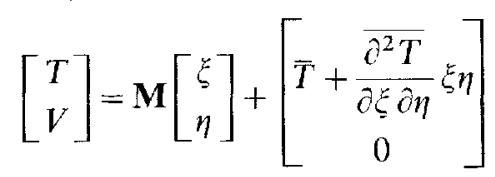

where $\mathbf{M}$ is the matrix of the transformation

$$
\mathbf{M}=\left[\begin{array}{cc}
\frac{\partial T}{\partial \xi} & \frac{\bar{\partial} T}{\partial \eta} \\
\frac{\partial T}{\partial T} & \frac{\partial T}{\partial \xi}
\end{array}\right]
$$

As we shall see later, this transformation solves many of the problems of the previous one. However, when the temperature varies linearly within the element, giving a straight interface, it would be elegant that this transformation becomes the identity. This may be achieved by writing

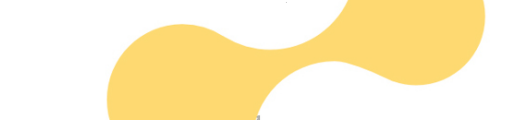

$$
\left[\begin{array}{c}
\zeta^{\prime} \\
\eta^{\prime}
\end{array}\right]=\mathbf{M}^{-1}\left[\begin{array}{c}
T-\bar{T} \\
V
\end{array}\right]
$$

where
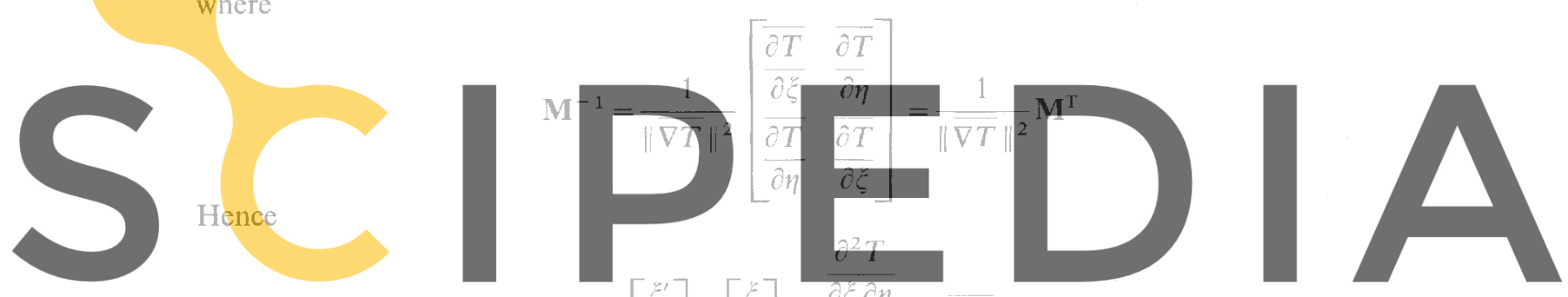

(11)

Register for free at https//www.scipedia.com to downtoad the version without the watermark

Equation (11) satisfies the previous requirements. First, for $\xi=$ constant equation (11) gives the parametric equation of one side of the quadrilateral in the $\left(\xi, \eta^{\prime}\right)$ plane with $\eta$ as the parameter. But $\xi^{\prime}$ and $\eta^{\prime}$ are linear functions of $\eta$ alone. The same holds for a side with $\eta=$ constant. Hence the transformation maps quadrilaterals with sides parallel to the axes $(\xi, \eta)$ onto quadrilaterals in the $\left(\xi^{\prime}, \eta^{\prime}\right)$ plane. Additionally, when the temperature varies linearly within the element, the second derivative in equation (10) is null and the transformation (11) becomes the identity. Furthermore multiplying equation (11) by $\nabla T$, we can see that every isotherm $T=T_{\mathbf{p}}$ is a straight line in the $\left(\xi^{\prime}, \eta^{\prime}\right)$ plane since

Denoting

$$
(\overline{\nabla T})^{\mathrm{r}}\left[\begin{array}{c}
\xi^{\prime} \\
\eta^{\prime}
\end{array}\right]=(\bar{\nabla} \bar{T})^{\mathrm{T}}\left[\begin{array}{l}
\xi \\
\eta
\end{array}\right]+\frac{\partial^{2} T}{\partial \xi \partial \eta}-\xi \eta=T_{\mathbf{P}}-\bar{T}=\mathrm{constant}
$$

$$
\begin{aligned}
& A_{\xi}=\frac{\overline{\partial T}}{\partial \xi} \frac{\overline{\partial^{2} T}}{\partial \xi \partial \eta} /\|\overline{\nabla T}\|^{2} \\
& A_{\eta}=\frac{\overline{\partial T}}{\partial \xi} \frac{\partial^{2} T}{\partial \xi \partial \eta} /\|\overline{\nabla T}\|^{2}
\end{aligned}
$$


equation (11) reduces to

$$
\left[\begin{array}{l}
\xi^{\prime} \\
\eta^{\prime}
\end{array}\right]=\left[\begin{array}{l}
\xi \\
\eta
\end{array}\right]+\left[\begin{array}{l}
A_{\xi} \\
A_{\eta}
\end{array}\right] \xi \eta
$$

We can take as a measure of the deformation of the element the coefficient

$$
A_{\xi}^{2}+A_{\eta}^{2}=\left(\frac{\overline{\partial^{2} T}}{\partial \xi \overline{\partial \eta}}\right)^{2} /\|\nabla \bar{T}\|^{2}
$$

which is the relation between the curvature and the temperature gradient. The determinant of the Jacobian $\mathbf{J}^{\prime}$ of this transformation reads

$$
\operatorname{det} \mathbf{J}^{\prime}=\left(1+A_{\xi} \eta\right)\left(1+A_{\eta} \xi\right)-A_{\xi} \xi A_{\eta} \eta=1+A_{\xi} \eta+A_{\eta} \xi
$$

From equation (13) we can see that the determinant of $\mathrm{J}^{\prime}$ is a linear function of $\xi$ and $\eta$ Therefore, for the Jacobian to be positive everywhere in the quadrilateral, it is necessary and sufficient that the Jacobian be positive at the four corners of the element. Thus this condition reads

$$
1 \pm A_{\xi} \pm A_{\eta}>0
$$

\section{which is equivalent to}
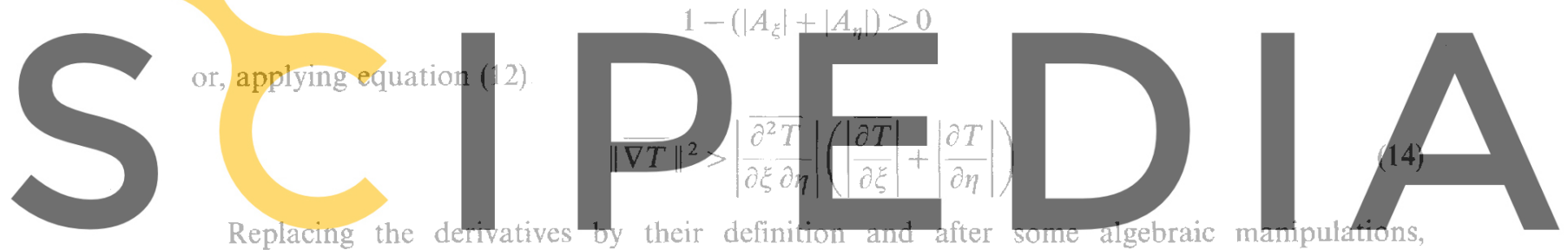

condition (14) reads

Register for free at https//www.scipedia.com to down $n_{\Delta_{1}}$ load the version without the watermark

where

$$
|\Delta|\left|\Delta_{2}\right|<\Delta_{1}^{2}+\Delta_{1}^{2}
$$

$$
\begin{aligned}
\Delta & =T_{1}+T_{3}-T_{2}-T_{4} \\
\Delta_{1} & =T_{3}-T_{1} \\
\Delta_{2} & =T_{2}-T_{4}
\end{aligned}
$$

Figure 5 shows the plot of these equations in the $\Delta_{1}, \Delta_{2}$ plane, the Jacobian being negative inside the area enclosed by the circles. The square plotted in the same Figure is defined by the equation

$$
\left|\Delta_{1}\right|+\left|\Delta_{2}\right|=|\Delta|
$$

whose interior represents the region where, for a given temperature, there are two isotherms going through the element. This occurs when

$$
\min \left(T_{1}, T_{3}\right)>\max \left(T_{2}, T_{4}\right) \text { or } \max \left(T_{1}, T_{3}\right)<\min \left(T_{2}, T_{4}\right)
$$

Thus taking a temperature $T^{*}$ such that $\min \left(T_{1}, T_{3}\right)>T^{*}>\max \left(T_{2}, T_{4}\right)$ or $\max \left(T_{1}, T_{3}\right)<$ $T^{*}<\min \left(T_{2}, T_{4}\right)$, the isotherm $T=T^{*}$ cuts the four sides of the quadrilateral as shown in Figure 6. We can see that the region of negative Jacobian almost coincides with the region 


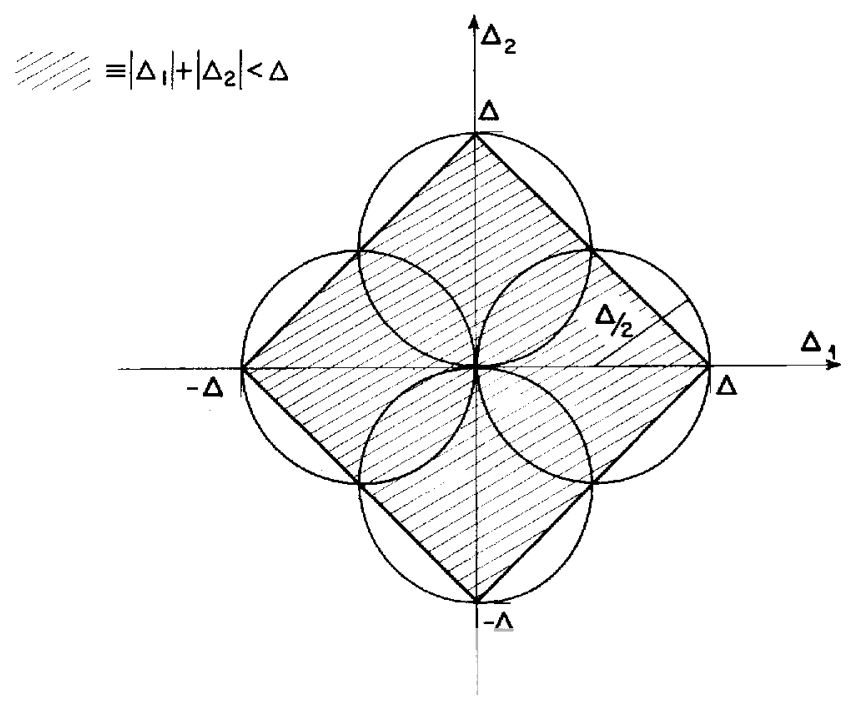

Figure 5. Analysis of the mapping. Region of negative Jacobian
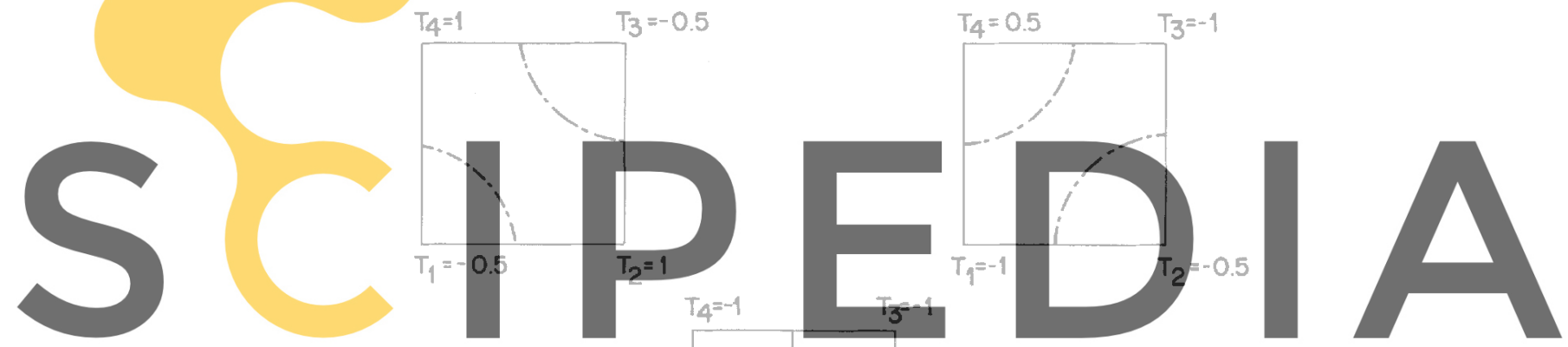

Register for free at https//www. scipedia.com to download the version without the watermark

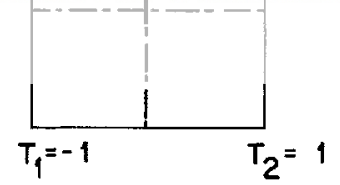

Figure 6. Sketch of two-phase elements traversed by two interfaces

where the two-phase element has two interfaces, but this situation is very rare in a numerical computation. Hence we can conclude that in a large number of problems the mapping (11) will behave adequately.

To verify the accuracy of this mapping, we can set $H_{1}-H_{\mathrm{s}}=1$, in equation (8b); obtaining

$$
\sum_{j}[\mathbf{i}]_{j}=\int_{\Omega_{1}^{n}} \sum_{j} N_{j} \operatorname{det} \mathbf{J} \mathrm{d} \xi \mathrm{d} \eta=\int_{\Omega_{1}^{n}} \operatorname{det} \mathbf{J} \mathrm{d} \xi \mathrm{d} \eta
$$

But this integral is equal to the area $A_{1}$ of the liquid region (the hatched area in Figure $7(a)$ ). This area can be calculated exactly by the expression

$$
A_{1}=2+\frac{1}{\beta^{2}}\left[2 \beta+\left(\beta^{2}-1\right) \ln \frac{1+\beta}{1-\beta}\right]
$$


where

$$
\beta=\frac{T_{3}+T_{1}}{T_{3}-T_{1}}
$$

The results given by the numerical and exact solutions are compared in Figure 7(b) as a function of $\beta$. The agreement is very good even when $\beta \cong 1$, i.e. for interfaces with high curvature.

It remains to analyse the ease of the inversion of co-ordinates, that is to go from $\left(\xi^{\prime}, \eta^{\prime}\right)$ to $(\xi, \eta)$. With some algebraic manipulations we can write

$$
A_{n} \xi^{2}+\left[1+\left(A_{\xi} \eta^{\prime}-A_{n} \xi^{\prime}\right)\right] \xi-\xi^{\prime}=0
$$

Dividing by $\xi^{2}$ and solving the quadratic equation in $1 / \xi$, we find

$$
\xi=2 \xi^{\prime} /\left[\left(1+A_{\xi} \eta^{\prime}-A_{\eta} \xi^{\prime}\right)+1+\left(A_{\xi} \eta^{\prime}-A_{\eta} \xi^{\prime}\right)^{2}+2\left(A_{\xi} \eta^{\prime}+A_{\eta} \xi^{\prime}\right)\right]
$$

Furthermore, the Jacobian of the transformation is

$$
\left|\frac{\partial\left(\xi^{\prime}, \eta^{\prime}\right)}{\partial(\xi, \eta)}\right|=1+\left(A_{\xi} \eta^{\prime}-A_{\eta} \xi^{\prime}\right)^{2}+2\left(A_{\xi} \eta^{\prime}+A_{\eta} \xi^{\prime}\right)
$$

Thus we can see that, at any Gauss point used for the numerical integration, we need to evaluate only one square root for the co-ordinate inversion and the computation of the Jacobian.

Finally, we shall devote a paragraph to comment on other options for solution. First, the interface might be considered straight, as in Reference 16. This method could give some errors

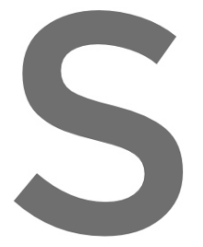
in the residual for highl into several straight line

Register for free at https//www.scipedia.com to download the version without the watermark

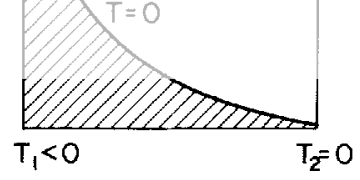

Figure 7(a). Integration over a hatched arca with a curved side

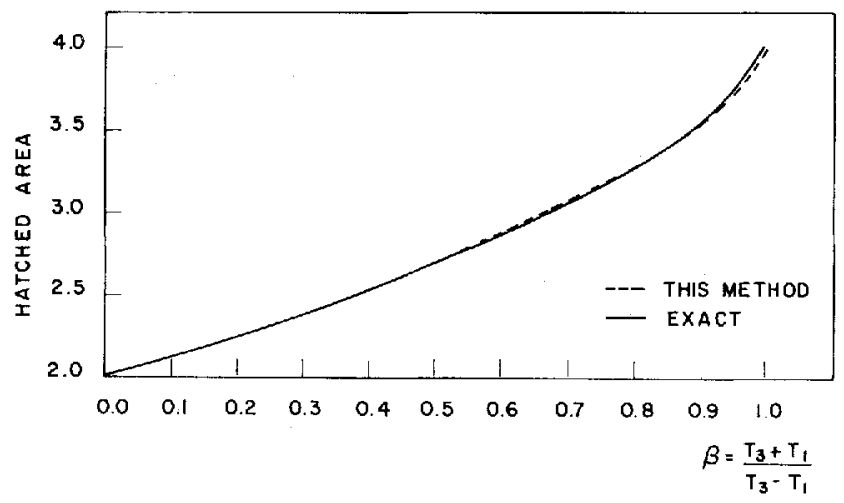

Figure $7(\mathrm{~b})$. Comparison between the numerical and exact values of the hatched area for several values of the parameter $\beta$ 
program than the simple one produced by the method proposed in this work. For instance, it is not simple to determine the number and location of straight line segments required, and the integration formulae become more involved. Another possibility is given by using linear triangles instead of linear quadrilaterals, because the triangles overcome the difficulties presented by the quadrilaterals since the linearly interpolated temperature gives rise to piecewise linear interfaces. However, the method presented in this paper is more general and may be adapted to elements with curved sides.

\section{THE INCREMENTAL SOLUTION ALGORITHM}

The next step is the solution of the non-linear system (4). This process may be viewed as finding a root of the residual

$$
\mathbf{r}(\mathbf{u})=\mathbf{K u}+\frac{1}{\Delta t} \mathbf{i}(\mathbf{u})-\mathbf{g}=0
$$

This is not an easy task owing to the step-like behaviour of the residual in this type of problem. Moreover when no special care is devoted to the solution scheme, the temperature in those nodes associated with phase-changing elements may jump above or below the melting value during the iterative process. The explanation of these oscillations is found in the amount of latent heat involved in each rearrangement of the interface poșition. Currently in a finite element context, the search process is achieved in an incremental form, i.e.

$$
\begin{aligned}
\mathbf{S} \Delta \mathbf{u}_{n}^{j} & =-\mathbf{r}\left(\mathbf{u}_{n}^{j}\right) \\
\mathbf{u}_{n}^{j+1} & =\mathbf{u}_{n}^{j}+\Delta \mathbf{u}_{n}^{j}
\end{aligned}
$$

where $\mathbf{S}$ is an appropriate iteration matrix, usually the Jacobian matrix of (15) and the superscript $j$ stands for the iteration number. However the residual in equation (15) is not differentiable and some skill must be used to obtain an adequate iteration matrix. The main duty of this matrix is to damp the spurious oscillations aforementioned. As the information of the interface movement is enclosed in the residual vector, one manner to accomplish this task is to appropriately include this vector in S. Quasi-Newton methods ${ }^{20}$ provide a systematic way to do this job. In Reference 16 an algorithm of this type is developed that makes a diagonal correction of $\mathbf{S}$ that has shown to be adequate to handle this type of instabilities. During the iterative process it is useful to use underrelaxation in the first iterations to enhance stability and overrelaxation to improve the convergence rate at the last ones. The relaxation parameter can be properly determined using a line search technique ${ }^{16}$ which determines the size of the increment which makes the projection of the residual in the search direction vanish, i.c. find $\sigma$ so that

$$
\left(\Delta \mathbf{u}_{n}^{j}\right)^{\mathrm{T}} \mathbf{r}\left(\mathbf{u}_{n}^{j}+\sigma \Delta \mathbf{u}_{n}^{j}\right)=0
$$

updating the unknown as

$$
\mathbf{u}_{n}^{j+1}=\mathbf{u}_{n}^{j}+\sigma \Delta \mathbf{u}_{n}^{j}
$$

Performing exact line searches satisfying equation (16) precisely is rather an expensive task. However, numerical experimentation has shown that it suffices to satisfy a less restrictive relation of the form

$$
\left|\frac{\left(\Delta \mathbf{u}_{n}^{j}\right)^{\mathrm{T}} \mathbf{r}\left(\mathbf{u}_{n}^{j}+\sigma \Delta \mathbf{u}_{n}^{j}\right)}{\left(\Delta \mathbf{u}_{n}^{j}\right)^{\mathrm{T}} \mathbf{r}\left(\mathbf{u}_{n}^{j}\right)}\right|<\varepsilon_{1}
$$


where the parameter $\varepsilon_{1}$ is set to, say, 0.9 .

To stop the iteration we measure the magnitude of the out-of-balance of the solution, that is iteration proceeds until

$$
\frac{\|\mathbf{r}(\mathbf{u})\|}{\|\mathbf{K u}\|}<\varepsilon_{\mathbf{R}}
$$

where $\varepsilon_{\mathrm{R}}$ is the required threshold for the normalized residual.

\section{NUMERICAL EXAMPLES}

The performance of the formulation described above has been examined in several numerical problems. In the following we present the solution for three examples. The first one concerns the solidification of a semi-infinite region defined by $x>0, y>0$. This problem is used as a benchmark to evaluate the accuracy of the method, since it is possible to obtain an analytic solution. ${ }^{21}$ The initial temperature is $0.3{ }^{\circ} \mathrm{C}$ and the half plane is frozen by lowering the temperature on the sides $x=0$ and $y=0$ to $-1^{\circ} \mathrm{C}$. The thermal properties are

$$
\begin{aligned}
k_{\mathrm{s}}=k_{1} & =1 \mathrm{kcal} / \mathrm{m} \mathrm{s}^{\circ} \mathrm{C}, & \rho_{\mathrm{c}_{\mathrm{s}}}=\rho_{\mathrm{c}_{1}} & =1 \mathrm{kcal} / \mathrm{m}^{3}{ }^{\circ} \mathrm{C} \\
\rho L & =2 \mathrm{kcal} / \mathrm{m}^{3}, & T_{\mathrm{m}} & =0{ }^{\circ} \mathrm{C}
\end{aligned}
$$

The finite element mesh used is depicted in Figure 8. Since the problem is symmetric about the line $x=y$, we restrict our analysis to the region $y>0 ; x>y$, imposing adiabatic conditions on the boundary $x=y$; i.e. side OA. To simulate the infinite region, adiabatic conditions have been imposed on the sides $\mathrm{AB}$ and $\mathrm{BC}$. Finally, the temperature $T=T_{\mathrm{w}}=-1{ }^{\circ} \mathrm{C}$ is imposed on side $\mathrm{OC}$. The 100 element mesh is refined near the origin to reproduce better the transient condition at $t=0$. The time step used is $0.01 \mathrm{~s}$. Figures 9 and 10 show the temperature profile along the line $x=y$ as a function of the distance to the origin $R$, for two time steps. The numerical solution is compared with the analytical solution of Budhia and Krieth. ${ }^{21}$ Figure 11 shows the time evolution of the interface position. The mean CPU time needed for computing the residual vector in the first time step was $6.158 \mathrm{~s}$. This is the time step when more elements change phase. For the same mesh in the linear case (without phase-change and discontinuous integration) the mean CPU time was $5.825 \mathrm{~s}$. For the straight-interface assumption the mean CPU time was $5.906 \mathrm{~s}$. The curved-interface method is 4 per cent more expensive than the straight-interface algorithm and 5.7 per cent more expensive than the linear case. Runs were performed on a VAX 11/780. The additional cost due to the new formulation is irrelevant.

The second example uses a coarser mesh for the same problem. The mesh depicted in Figure 12 uses 100 equally spaced elements to discretize the $90^{\circ}$ corner region of the half plane. The time step used in this example is $0.02 \mathrm{~s}$. Figures 13 and 14 show the temperature profile along the line $x=y$ for two different time steps. In Figure 15 the interface locus is plotted for $t=0.04 \mathrm{~s}$.

The third example involves an internal heat source. The temperature distribution is given by

where

$$
T= \begin{cases}2 f(x, y, t), & f>0 \\ 1.5 f(x, y, t), & f<0\end{cases}
$$

$$
f=R+0 \cdot 25 t-1
$$

$R$ being the distance measured from the point $\mathrm{P}=(0 \cdot 1,0)$. The interface locus is an arc of circumference at any time. It is represented by the equation

$$
R=1-0.25 t
$$




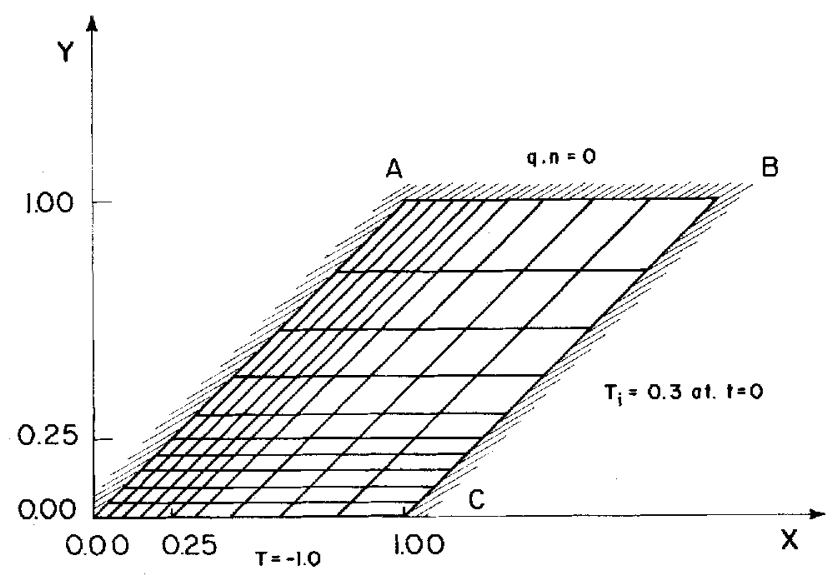

Figure 8. Model problem 1. Finite element mesh

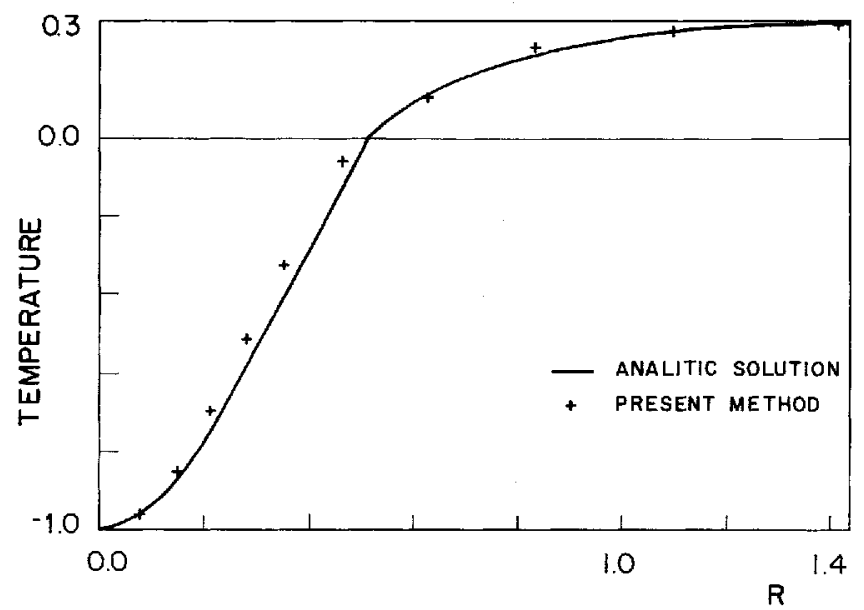

Figure 9. Model problem 1. Temperature distribution for lime $=0.04 \mathrm{~s}$. Analytical $(-)$ and numerical (+) solutions

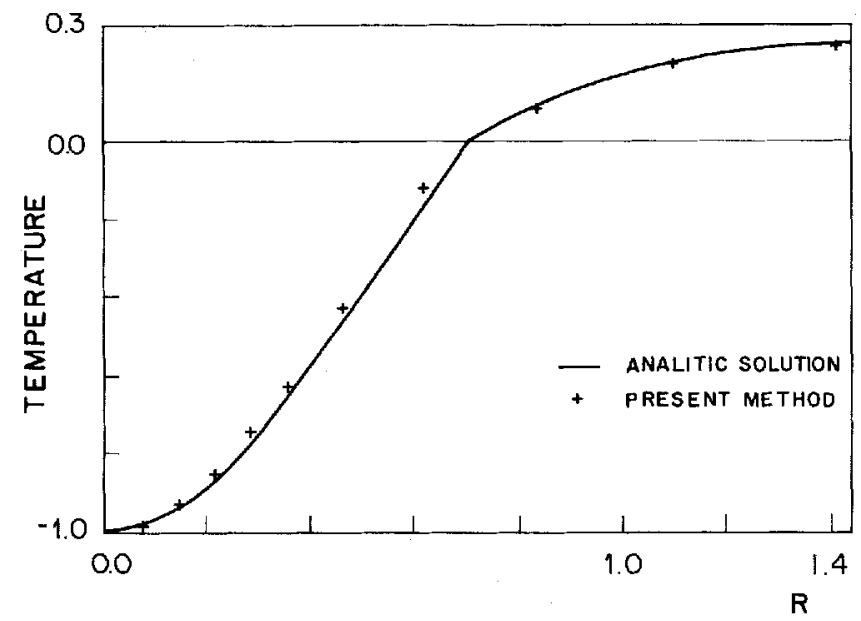

Figure 10. Model problem 1. Temperature distribution for time $=0.08 \mathrm{~s}$. Analytical $(-)$ and numerical $(+)$ solutions 


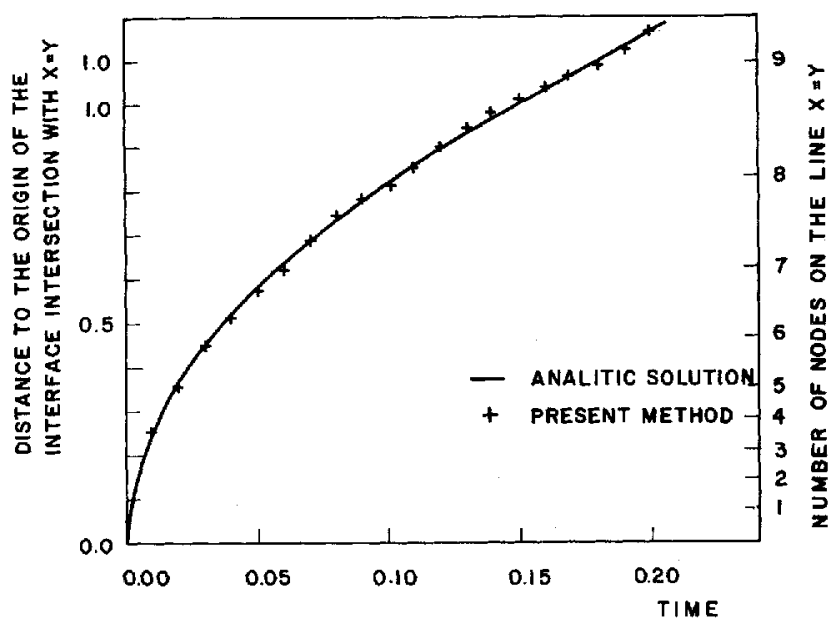

Figure 11. Model problem 1. Evolution of the front position. Analytical ( -$)$ and numerical $(+)$ solutions

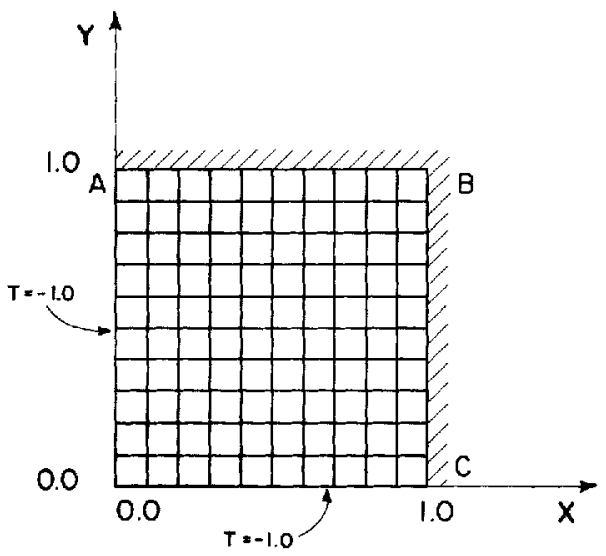

Figure 12. Model problem 2. Finite element mesh

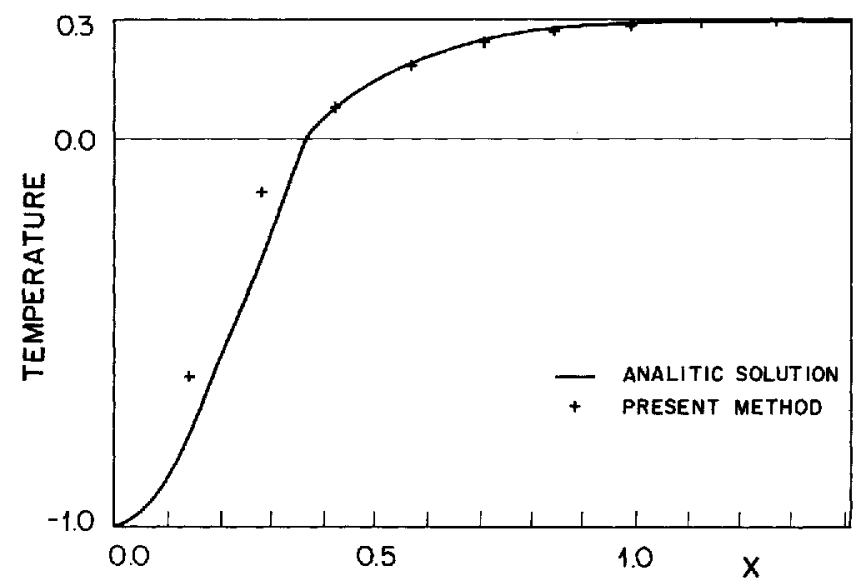

Figure 13. Model problem 2. Temperature distribution for time $=0-04 \mathrm{~s}$. Analytical $(-)$ and numerical $(+)$ solutions 


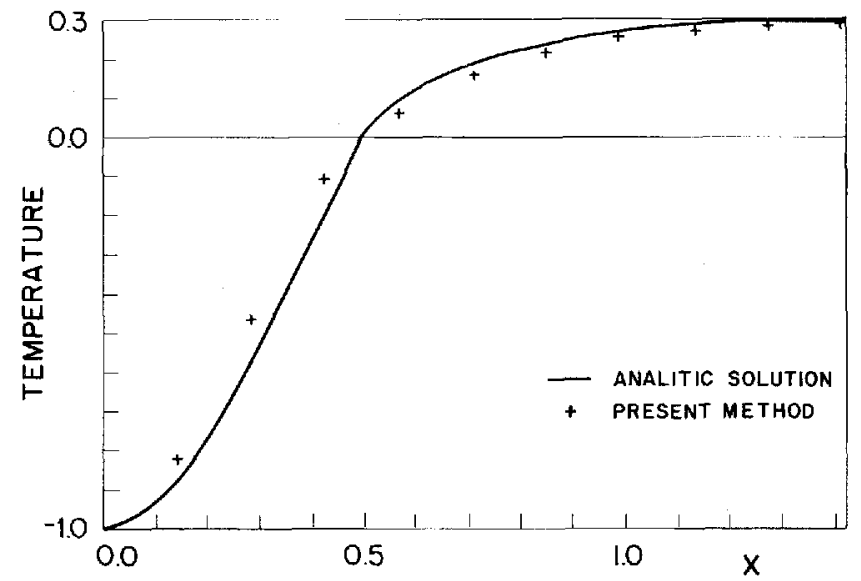

Figure 14. Model problem 2. Temperature distribution for time $=0 \cdot 08$ s. Analytical $(-)$ and numerical $(+)$ solutions

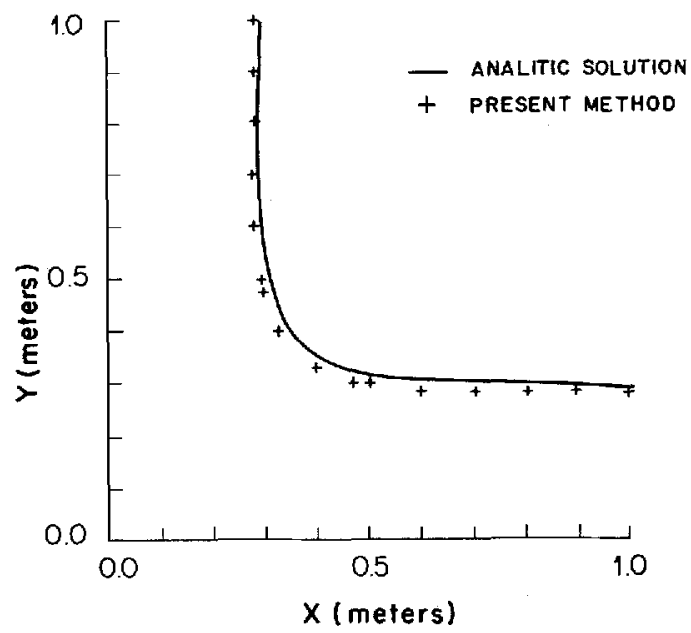

Figure 15. Model problem 2. Interface location for time $=0.04 \mathrm{~s}$. Analytical $(-)$ and numerical $(+)$ solutions

and thus its normal is the radial unit vector. The thermal properties are the same as for the first examples.

This temperature distribution is achieved by using the internal heat source given by ${ }^{22}$

$$
Q_{(x, y, z)}= \begin{cases}2\left(0.25-\frac{1}{R}\right), & f>0 \\ 1 \cdot 5\left(0.25-\frac{1}{R}\right), & f<0\end{cases}
$$

and the corresponding boundary conditions arising from equation (17).

Figure 16 depicts the 272 finite-element mesh used for the discretization of the region enclosed by the line ABCDEFGOA. On the whole boundary the temperature is imposed according to (17). The time step used is $025 \mathrm{~s}$. Figure 17 plots the interface position for two time steps. Figure 18 shows the temperature profile at $t=1 \mathrm{~s}$ for several lines of constant $y$. 


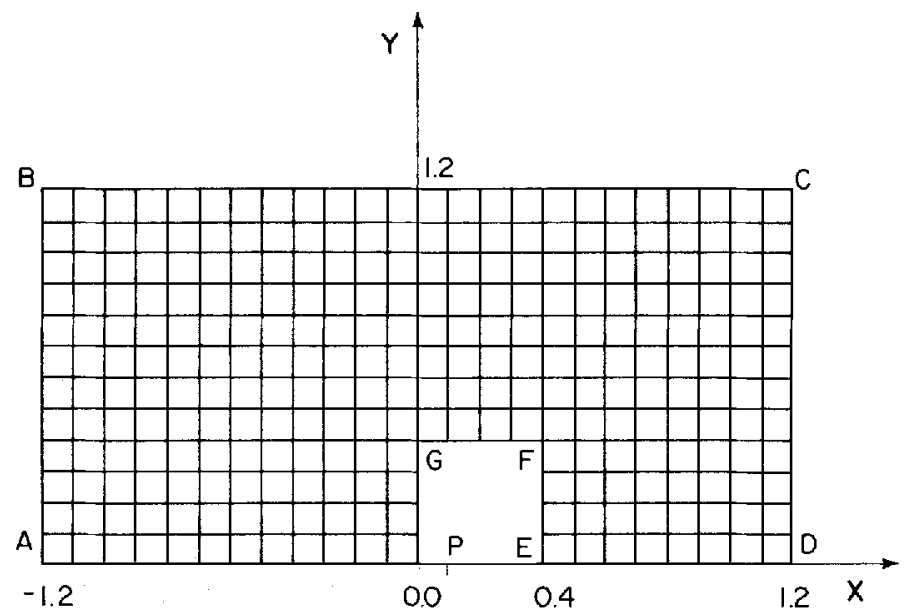

Figurc 16. Model problem 3. Finite element mesh

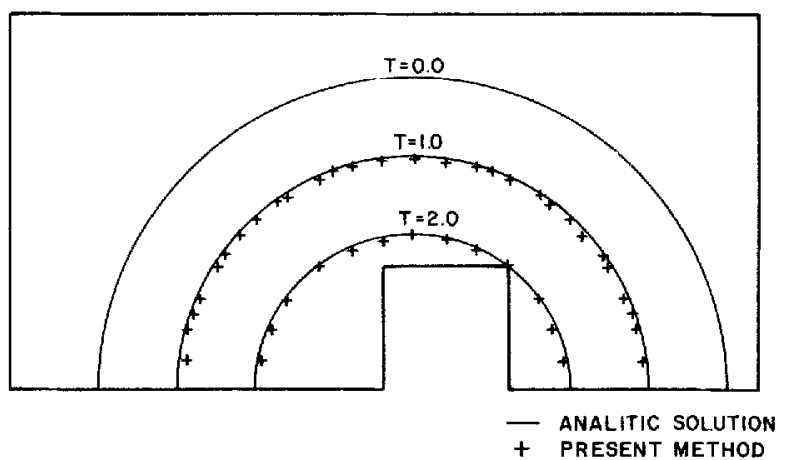

Figure 17. Model problem 3. Interface location for several time stcps. Analytical (-) and numerical $(+)$ solutions

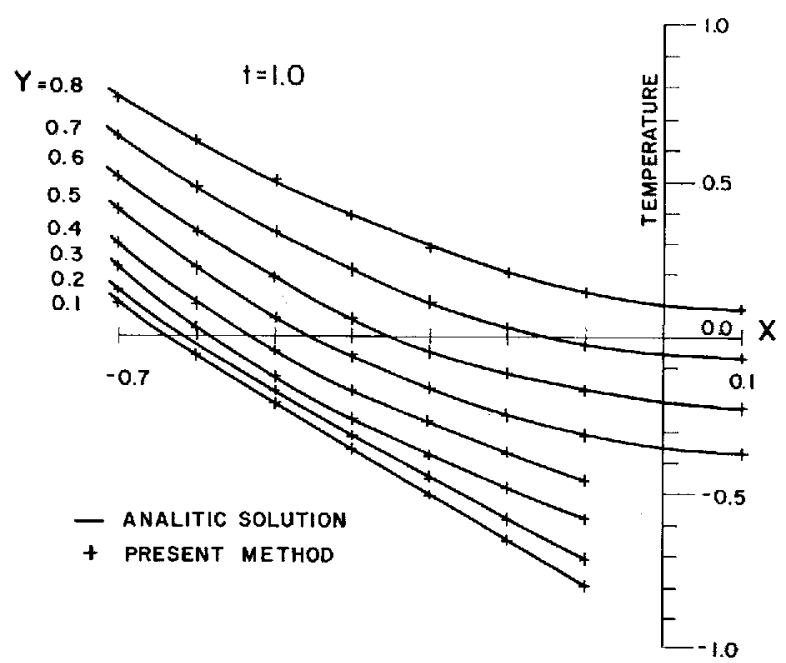

Figure 18. Model problem 3. Temperature distribution for time $=1 \mathrm{~s}$. Analytical $(-)$ and numerical $(+)$ solutions 


\section{CONCLUSIONS}

A new procedure for dealing with curved interfaces has been presented. It allows an improved integration of discontinuous functions within the elements which are traversed by such interfaces. The procedure has been applied to model two-dimensional phase-change problems. The solution is obtained in terms of temperature, without the current requirement of an explicit smoothing of the temperature-enthalpy relation. The algorithm features an accurate integration of the enthalpy contribution to the residual vector. Furthermore, the convergence criterion is governed by the norm of this residual vector.

The iteration process is handled by a careful strategy to avoid spurious oscillations and lack of convergence. It combines a proper modification of the iteration matrix with an approximate line search.

This algorithm may be readily implemented in finite element packages without any major modification of the program. Little additional calculation is required to evaluate the proposed transformation, only a square root at each Gaussian point is needed to compute the Jacobian and to invert the co-ordinate system. The computation of the integrals in those elements traversed by the interface does not produce relevant extra cost. The method behaves encouragingly in the examples performed and our experience indicates that it is possible to use coarser meshes and larger time steps than used by current fixed-domain methods. Notwithstanding, theoretical studies concerning the rate of convergence of the method are still needed.

\section{ACKNOWLEDGEMENTS}

M. Storti and L. A. Crivelli would like to thank the Consejo Nacional de Investigaciones Cientificas y Tecnicas (CONICET), Argentina, for the support given via a research fellowship provided for this work.

\section{REFERENCES}

1. A. Fasano and M. Primicerio, Free Boundary Problems: Theory and Applications, Pitman Advanced Publishing Program, London, 1983.

2. J. R. Ockendon and W. R. Hodgkins (cds), Moving Boundary Problems in Heat Flow and Diffusion, Oxford University Press, Oxford, 1975.

3. L. I. Rubenstein, The Stefan Problem, American Mathematical Society, Providence, 1971.

4. K. O'Neill and R. Lynch, 'A finite element solution for freezing problems, using a continuously deforming coordinate system', in, R. W. Lewis, K. Morgan and O. C. Zienkiewicz (eds), Numerical Methods in Ileat Transfer, Wiley, New York, 1981.

5. D. Lynch, 'Unified approach to simulation on deforming elements with application to phase change problems', $J$. Computational Physics, 47, 387411 (1982).

6. M. Hogge and P. Gerrekens, 'Two-dimensional deforming finite element methods for surface ablation', AIAA 18th Thermophysics Conference, Montreal, Canada, 1-3, June 1983.

7. K. O'Neill, 'Boundary integral equation solution of moving boundary phase change problems', Int. j. numer. methods eng., 19, 1825-1850 (1983).

8. C. M. Elliot and J. R. Ockendon, Weak and Variational Methods for Moving Boundary Problems, Pitman, Boston, 1982.

9. D. G. Wilson, A. D. Solomon and P. T. Boggs, Moving Boundary Problems, Acadcmic Press, New York, 1978.

10. D. R. Atthey, 'A finite difference scheme for melting problems', J. Inst. Math. Applics., 13, 353- 366 (1974).

11. G. Comini, S. Del Guidice, R. W. Lewis and O. C. Zienkiewicz, 'Finite element solution of non-lincar heat conduction problems with special reference to phase change', Int. $j$. mumer. methods eng., 8, 613-624 (1974).

12. G. H. Meyer, 'Multidimensional Stefan problems', Siam J. Numer. Anal., 10, 522-538 (1973).

13. W. D. Rolph III and K. J. Bathe, 'An efficient algorithm for analysis of nonlinear heat transfer with phase changes', Int. j. numer. methods eng., 18, 119-134 (1982).

14. J. Roose and O. Storrer, 'Modelization of phase changes by fictitious heat flow', Int. j. numer. methods eng., 20, 217-225 (1984).

15. D. Blanchard and M. Fremond, 'The Stefan problem: computing without the free boundary', Int.j. numer. methods eng., 20, $757-771(1984)$. 
16. L. Crivelli and S. Idclsohn, 'A temperature-based finite element solution for phase-change problems', Int. $j$. numer. methods eng., 23, 99-119 (1986).

17. G. P. Steven, 'Internally discontinuous finite elements for moving interface problems', Int. j. numer. methods eng., 18 , $569-582(1982)$.

18. O. C. Zienkiewicz, The Finite Element Method, 3rd edn, McGraw-Hill, London, 1977.

19. T. J. R. Hughes, 'Analysis of transient algorithms with particular reference to stability behaviour', in T. Belytschko and T. J. R. Hughes (cds), Computational Methods for Transient Analysis, Vol. 1, Elsevier Science Publishers, Amsterdam, 1983.

20. M. Geradin, M. Hogge and S. Idelsohn, 'Implicit finite element methods', in T. Belytschko and T. J. R. (eds), Computational Methods for Transient Analysis, Vol. 1, Elscvicr Scicncc Publishers, Amsterdam, 1983.

21. H. Budhia and F. Krieth, 'Heat transfer with melting or freezing in a wedge', Int. J. Heat Mass Transfer, 16, 195-211 (1973).

22. R. H. Nochetto, 'Analisis numerico del problema de Stefan multidimensional a dos fases por el metodo de regularizacion', Tesis Doctoral, Universidad Nacional de Buenos Aires, 1982. 